\title{
Lecturas y relatos históricos de la Terapia Ocupacional en Suramérica. Una perspectiva de reflexión crítica ${ }^{1}$
}

\author{
Readings and historical accounts of Occupational Therapy \\ in South America. A critical reflection perspective
}

\author{
Alejandro Guajardo Córdoba
}

Recibido: 13 de junio 2016 • Enviado para modificación: 22 de agosto 2016 • Aceptado: 20 de noviembre 2016

Guajardo, A. (2016). Lecturas y relatos históricos de la Terapia Ocupacional en Suramérica. Una perspectiva de reflexión crítica. Revista Ocupación Humana, 16 (2), 110-117.

\begin{abstract}
Resumen: Esta reflexión propone originalmente a la Terapia Ocupacional como un dispositivo tecnológico (profesión) de carácter histórico y social. Su desarrollo y devenir deben ser comprendidos y explicados en su estrecha relación con la historia social y política de la región latinoamericana. El fundamento originario de concepción anglosajona ordena y domina los conceptos y métodos de nuestra praxis en las primeras décadas, esto pone al oficio en la región en un orden subalterno. Desde una posición crítica, se debate la necesidad de construir una historia propia, descolonizada, desde el Sur, que sitúe el quehacer disciplinar y profesional en estrecha vinculación con los problemas sociales, económicos y culturales que afectan a los grandes grupos humanos excluidos; asimismo, el desafío ético de una Terapia Ocupacional comprometida con la humanización y la libertad humana. De otro lado, se problematiza la expansión del oficio bajo el alero de políticas públicas y sociales, y su estrecha implicación de carácter biopolítico; también, la manera en que se moldean sus objetivos, conceptos y métodos. Finalmente, se propone una Terapia Ocupacional crítica, ética, fundamentada en los derechos humanos, reflexiva, autoconsciente y permanentemente en rebeldía consigo misma y con el contexto social.
\end{abstract}

Palabras clave: Historia, Terapia Ocupacional, América del Sur, ciencia y sociedad

Abstract: This reflection proposes originally occupational therapy as a technological device (profession) of historical and social character. Its development and evolution must be understood and explained in its close relation with the social and political history of the Latin American region. The original foundation of Anglo-Saxon conception leads and dominates the concepts and methods of our praxis in the first decades of occupational therapy and this places our profession in the region as a subordinate of the Anglo-Saxon world. From a critical standpoint, the need to construct our own, Southern, decolonized history is set, to place the disciplinary and professional work closely linked to the social, economic and cultural problems that affect the large excluded human groups; and the ethical challenge of an occupational therapy committed to humanization and human freedom. Also, the problematization of the expansion of the profession under the auspices of public and social policies, its close

\footnotetext{
${ }^{1}$ Este artículo se basa en la ponencia presentada por el autor el 3 de marzo de 2016 en el panel inaugural del XVI Congreso Colombiano de Terapia Ocupacional celebrado en Medellín, Colombia.

${ }^{2}$ Terapeuta Ocupacional. Especialista en Derechos Humanos y Discapacidad. Profesor y Jefe Carrera de Terapia Ocupacional. Universidad de Santiago de Chile. Observatorio de Derechos Humanos para Personas con Discapacidad Mental. Santiago de Chile, Chile. alejandro.guajardo@usach.cl
} 
relationship to the biopolitical nature, and the way in which its objectives, concepts and methods are shaped, is presented. Finally, I propose a critical, ethical occupational therapy, based on human rights, reflexive, self-conscious and in permanent rebellion with itself and the social context.

Key words: History, occupational therapy, South America, science and society

\section{Introducción}

Ya nos dice Bachelar (Briones, 2006) que la historia constituye un aspecto central para reflexionar cualquier tema en ámbitos como la ciencia, la cultura, lo social. Ello previene de asumir la realidad, las ideas, los conceptos de manera abstracta y naturalizada. El análisis histórico es una condición obligada para cualquier reflexión, pues nos remitirá siempre a una sociedad concreta y determinada.

La existencia de la Terapia Ocupacional no es un hecho de carácter individual o personal. Tampoco es un hecho natural que radique en el ámbito de las ciencias naturales. La Terapia Ocupacional no es una práctica exterior a la realidad humana, a la historia humana. La Terapia Ocupacional es una producción histórica.

De esta manera, la revisión y la reflexión sobre nuestro oficio y disciplina desde una lógica histórica resultan trascendentes, pues darán cuenta de las condiciones objetivas, materiales, políticas, económicas, culturales y subjetivas que nos pueden describir, hacer comprender y explicar la existencia de la profesión.

Así, la reflexión histórica por los cincuenta años se transforma en un imperativo necesario, de orden político, comprendiendo esta noción -lo político- como el lugar del bien común, de lo público y del interés colectivo, lugar de reconocimiento de que todos somos lo mismo.

\section{Acerca de la historia y su relación con lo político}

Toda narrativa de la historia institucional es el discurso de nosotros mismos sobre nosotros mismos bajo determinadas condiciones históricas. Es decir, es un sujeto que habla sobre el sujeto. La historia es el territorio producido por nosotros, territorio que vivimos y habitamos (Martínez, 2011).

Así, referirse a las condiciones históricas en las que la Terapia Ocupacional es producida es no compartir la visión de que esta ha evolucionado. Un enfoque evolucionista alude a un desarrollo, a una continuidad, a un devenir, ir de un punto a otro, una meta a la cual llegar y alcanzar. Ir desde lo simple a lo complejo, desde una mirada acumulativa de conocimientos y experiencias, deshistorizada y fuera de las condiciones sociales que han producido dispositivos tecnológicos, como la Terapia Ocupacional, es la concepción cientificista de orden positivista de la historia.

Por el contrario, una lectura historizada entenderá la Terapia Ocupacional 
como un dispositivo social que tiene un origen suprapersonal y está más allá de los individuos y de las relaciones microsociales. Comprenderá que la Terapia Ocupacional, así como otras profesiones, son el resultado del modelo de desarrollo político, social y del grado alcanzado por una sociedad en particular por la forma en que organiza la producción material y simbólica, por la organización del trabajo, por las relaciones entre clases sociales, por la complejidad social alcanzada, por la distribución de la riqueza social (Vidal 1990), en síntesis, por los procesos de reproducción social.

Entonces, cabe preguntarse icuáles fueron las condiciones históricas que hicieronvg emerger la Terapia Ocupacional?

Desde la perspectiva señalada, podemos decir que la aparición y la fundación de la Terapia Ocupacional en Suramérica se dio en el escenario de un Estado protector y desarrollista, el cual, bajo la premisa de la universalidad, debía dar respuestas sociales amplias y abarcadoras a diferentes problemáticas de orden social, más aún luego de la Segunda Guerra Mundial y ante la necesidad de restablecer las economías del planeta sobre la base de un papel más activo del Estado. Así mismo, en lo particular en la región se sumaban las condiciones socio-sanitarias derivadas de las grandes epidemias de poliomielitis.

Alrededor de 1960 se generaron las condiciones para los cursos iniciales de Terapia Ocupacional. Ese período se caracterizó por grandes debates políticos asociados a proyectos de transformación social y por la disputa de modelos de desarrollo a impulsar en todos los países de la región; por ejemplo en Chile, la vía chilena al socialismo. A nivel internacional, marcaban de modo significativo a la región latinoamericana la revolución cubana y la contrapropuesta, desde los Estados Unidos, de la Alianza para el Progreso. Esta última, como sustrato de orden macro social, generó condiciones para la articulación de entidades como la Organización Panamericana de la Salud, ministerios de salud y universidades para la puesta en marcha de los primeros cursos de Terapia Ocupacional en la región. Se trataba de mostrar que no eran necesarias nuevas revoluciones de izquierda, y que Estados capitalistas, liberales y socializantes podían resolver los nuevos problemas emergentes (Guajardo, 2014).

En este acto fundacional también se instalaron determinadas formas de ver el mundo, la práctica social, la actividad humana y al sujeto. Nos referimos a la mirada que nosotros llamamos anglosajona, de carácter pragmático, cientificista, positivista, de países ricos, traducida en perspectivas funcionalistas de la Terapia Ocupacional. Como profesiones fuimos producidos y $\mathrm{CO}-$ lonizados cognitiva y subjetivamente desde este lugar.

Como he planteado, la Terapia Ocupacional tiene un origen claramente político y no técnico, y su masificación y consolidación como profesión se entreteje muy fuertemente al desarrollo de las políticas públicas y sociales. Las políticas públicas no solo han sido el contexto material a través del cual se despliegan el actual modelo 
neoliberal y la sociedad de mercado, también han articulado y configurado técnicamente las prácticas interventivas implementadas en la gran mayoría de las profesiones; la Terapia Ocupacional no ha sido ajena a ello. Es decir, las políticas públicas han operado de modo significativo sobre los aspectos técnicos y conceptuales de las acciones en Terapia Ocupacional.

La Terapia Ocupacional en Chile, a corto andar de su fundación (1963 primer curso, 1972 la primera carrera), quedó subsumida en la dictadura cívico - militar. Su instalación como nueva profesión se dio en el marco de las intervenciones institucionales, de políticas públicas centradas de modo ahistórico en el individuo frente a problemáticas médicas específicas. En este contexto la Terapia Ocupacional asumió un carácter claramente tecnocrático, sistémico funcionalista, que dicotomizó y desconoció los aspectos estructurales que la sustentaban; se desplegó en los grandes hospitales y, en menor medida, en las instituciones educativas y penitenciarias.

En el periodo posdictatorial (1990 a la fecha), la Terapia Ocupacional comienza a ser configurada por los modelos de desarrollo social en curso, introduciéndose con los años, con mayor sofisticación cada vez, en situaciones sociales definidas como deficiencias o carencias de determinadas personas o grupos. Los pasos de la Terapia Ocupacional en Chile comienzan a ser marcados por objetivos como la integración social, la pobreza, la reinserción de menores o personas con enfermedades mentales, la prevención del consumo de drogas y la violencia, entre otras. Esto conlleva una obligada diversificación de las praxis profesionales y una ruptura con la exclusividad del campo clínico de orden biomédico.

Así, la Terapia Ocupacional se ve interpelada a incorporar conocimientos y tecnologías sobre lo social, la planificación estratégica, las políticas sociales, el desarrollo social y la intervención social y comunitaria.

Adquiere importancia en las estrategias de intervención la categoría de lo social, con variables como género, territorialidad, identidad y, en los años 2000, fuertemente con el enfoque de Derechos Humanos. Se abordan grupos excluidos, en riesgo y vulnerados. Todo un nuevo campo discursivo y de praxis que da forma a la Terapia Ocupacional de los años noventa en adelante. Esto se traduce en la utilización de fuertes componentes de modelos centrados en las competencias, las redes sociales, los cuales se articulan muy bien con el enfoque de rehabilitación psicosocial en salud mental y la distribución de recursos para acceder a la rehabilitación en comunidad, al empleo y a la educación.

Habiéndome referido a Chile, considero que este proceso ha sido propio también de toda la región.

Desde la política pública y la política social se han abordado nuevas problemáticas sociales. Aquí la Terapia Ocupacional ha encontrado su expansión y consolidación en los diversos países, promoviendo su diversificación en múltiples tipos de prácticas. Es la vinculación de la Terapia Ocupacio- 
nal no con la enfermedad, sino con la cuestión social. Hemos transformado los problemas en objetos sociales de la Terapia Ocupacional, de estudios, investigación y construcción de modelos operativos. De esta forma, aparentemente la intervención predominante ya no es individual en el contexto médico clínico, sino más bien de grupo: los talleres, los grupos psicosociales, la familia, la situación escolar, la situación laboral, la participación en comunidad, entre otros. Esto ha generado un profundo cambio en el perfil profesional. Buena cuenta de ello lo encontramos en los interesantes e importantes desarrollos de la llamada Terapia Ocupacional Social en Brasil (Esquerdo, Malfitano, Silva \& Borba, 2015).

Sin embargo, lo que creo que ha estado sucediendo no es más que la terapeutización (asumo que es un neologismo) en los ámbitos más variados de la actividad humana, pero muchas veces sostenidos con la misma lógica de la intervención clínica biomédica positivista. Más que introducir modos de intervención más abiertos y horizontales, lo que se hace en la vida diaria concreta de los sujetos, en el espacio de vida particular (calle, casa, territorio), es generalizar la lógica de la terapia médica a situaciones que guardan relación con la vida social y política, transformado la vida cotidiana en verdaderas terapias del mundo de la vida.

La terapeutización del contexto social convierte en problemas individuales a los fenómenos de orden social: el agobio de la competitividad, la explotación, el sufrimiento. Convierte la crisis social del capitalismo neoliberal y de mercado en problemas subjetivos a tratar terapéuticamente. Convierte las aprensiones e inseguridades que derivan de la discriminación, la pobreza, la violencia y la soledad en índice de problemas internos, de dificultades cognitivas o comunicacionales, en problemas de destrezas y habilidades sociales, en problemas de autoestima y de capacidad de logro. La ausencia de acceso a derechos humanos y vida digna en deficiencias personales para la construcción del proyecto de vida.

\section{Desafíos para la profesión y la disciplina en los escenarios actuales}

¿Cómo desarrollar la profesión y la disciplina en la sociedad contemporánea?

El desafío histórico que interpela a la Terapia Ocupacional en estos cincuenta años conlleva fijar una posición ética y política de su fundamento y hacer. No es posible la neutralidad en una profesión cuyo centro es la actividad social manifestada como ocupación y materializada en sujetos que se encuentran en situación de exclusión, privación o segregación.

Implica una ruptura con el sustrato naturalista darwiniano, liberal del sujeto, a su manifestación en la ocupación como exterioridad del mismo y como única vía de mediación con el entorno. Se requiere de un quiebre con el cientificismo, la medición objetiva, los modelos operativos y de la práctica ajenos a nuestra realidad social y cultural. La exigencia es asumir una postura crítica, decolonizadora, donde esté en juego no solo lo referido a la clase 
social, la marginalidad y la exclusión, sino también el tema cultural, identitario, de género, el indigenismo, entre otros. Ser autores, con nuestra propia voz, de los procesos que nos atañen y nos convocan. Con la producción de saberes regionales, en permanente diálogo con escenarios situados en los países de Latinoamérica.

\section{A modo de síntesis y propuesta}

Somos el acto de habla de una sociedad inequitativa. Existimos porque hay dolor y sufrimiento. La expansión del oficio se debe a que cada vez hay más problemas sociales, mayor deshumanización, mayor cosificación de las personas. Nuestra autonomía como profesión es relativa, pues no estamos fuera de la realidad que nos ha producido. Somos el acto de habla de los espacios de poder. Ser un acto de habla es constituir un conjunto de enunciados, discursos (en ellos prácticas) que conforman y producen la realidad en el mismo momento del acto de enunciar.

Las prácticas de la Terapia Ocupacional, así como sus fundamentos teóricos, producen la realidad que enuncian. El ahistoricismo, el individualismo metodológico, nociones como ocupación en un sentido abstracto y naturalista, lo intrínseco y biológico como procesos del ser, entre otros, reproducen el actual orden social.

Lo que se requiere es una Terapia Ocupacional que transforme, que promueva otras formas de relaciones sociales, otras formas de vida. Es decir, dejar de ser la Terapia Ocupacional que somos para ser otra Terapia
Ocupacional. Una que promueva la democracia, el bien común y no el sistema social en el cual se ha sostenido. A esta Terapia Ocupacional la llamaré Terapias Ocupacionales desde el Sur (Guajardo, 2016).

Lo anterior exige asumir una posición crítica explícita para los diferentes ámbitos de la práctica profesional e investigativa, sea esta en salud, educación, justicia, protección social. Así mismo en la intervención clínica, psicosocial y comunitaria.

En palabras de Foucault (1995), la crítica es el arte de no ser gobernado, como una manera de desconfiar, poner en duda, cuestionar y transformar aquellas prácticas que conforman el arte de gobernar. El foco de la crítica, según este autor, está en las relaciones existentes entre poder, verdad y sujeto, en sus palabras: "la crítica es el movimiento por el cual el sujeto se atribuye el derecho de interrogar a la verdad acerca de sus efectos de poder, $y$ al poder acerca de sus discursos de verdad, pues bien, la crítica será el arte de la inservidumbre voluntaria, el de la indocilidad reflexiva" (p. 8).

Recurriendo a Georg Luckács (1972), debemos dejar de ser una profesión enajenada, un sujeto en sí -comprendiendo esto como una praxis tecnocrática, cientificista, reproductora del sistema social-, para transformarnos en un sujeto para sí, es decir, en una comunidad consciente de su posición y su situación histórica, de su condición de clase y de las personas que la conforman. Que la Terapia Ocupacional se asuma para sí implica, entre otras cosas, la politización de la pro- 
fesión; lo contrario, la despolitización de nuestra praxis, se traducirá en una ausencia de autoconciencia hacia lo que podríamos denominar noción de mundo en general, y de reconocernos como actores productores y reproductores del mundo de la vida.

La escasa formación política de profesiones de la salud, entre ellas la Terapia Ocupacional, y la excesiva racionalidad técnica de las profesiones, centradas en el método y el saber cientificista, no permiten advertir en ellas visiones y epistemes disidentes, resistentes al sistema, que generen puntos de fuga a los procesos de dominación que la Terapia Ocupacional estándar promueve y al régimen social neoliberal actual en el cual se inserta. Por el contrario, precisamente, la Terapia Ocupacional se extiende aquí como disciplina que funcionaliza sus prácticas sociales, incentivando la adaptación pasiva y privatizada de las personas y los colectivos, lo que se inserta organizadamente en los engranajes micro y macro políticos del sistema actual de dominación.

Las Terapias Ocupacionales disponen de una posibilidad que otras profesiones no tienen, pues se vincula y se remite siempre, como lo hemos señalado, al mundo de la vida, a la acción concreta sobre las personas, a las ocupaciones cotidianas, a colectivos y comunidades y a sus circunstancias históricas. Remite siempre a la praxis concreta, lo que abre la posibilidad de interrogarse: ¿Qué es lo que se pue- de hacer? ¿Cómo propiciar una mejor vida basada en los derechos fundamentales? ¿Cómo, desde la crítica y en contra de la despolitización de los oficios, puedo contribuir a una vida más digna de las comunidades y personas con las que la Terapia Ocupacional interactúa?

El valor que tienen las Terapias Ocupacionales desde el Sur como manifestación de diversidad de la disciplina y la profesión (Terapia Ocupacional Política, Terapia Ocupacional Social, Terapia Ocupacional Ecosocial, Terapia Ocupacional y Derechos Humanos, Terapia Ocupacional e Indigenismo), situadas desde las ciencias humanas, es la posibilidad de desarrollar prácticas más allá de la reproducción de dispositivos de salud, sociales, jurídicos y de administración de los sujetos. Es tener la posibilidad de reconstruir a través de las prácticas ocupacionales el sentido de lo público, de promover prácticas deliberantes de las/os ciudadanos, promover una vida digna para un habitar de lo social común en la diferencia, promover una vida donde la ocupación, que no es más que el sujeto mismo, se fundamente y se sustente en una ética basada en los derechos humanos ${ }^{3}$.

\section{Referencias}

Briones, G. (2006). Teorías de las Ciencias Sociales y de la Educación. Epistemología. México: Trillas.

Esquerdo R., Malfitano A., Silva C., \& Borba P. (2015) Historia, conceptos y propuestas en la Terapia Ocupacional Social en

\footnotetext{
${ }^{3}$ Referencia del profesor Iván Torres en relación a los desafíos que tiene la Terapia Ocupacional con respecto a los Derechos Humanos. Procesos de supervisión de seminarios de títulos con estudiantes de Terapia Ocupacional, 2015.
} 
Brasil. Revista Chilena de Terapia Ocupacional, 15 (1), 73-84.

Foucault, M. (1995). ¿Qué es la crítica? Crítica y Aufklarung. Revista de Filosofía, (11), 5-25.

Guajardo, A. (2014). Terapia Ocupacional. Apuntes para una historia inconclusa. En V. Do Santo \& A. Donatti (Eds.), Cuestiones contemporáneas de la Terapia Ocupacional en Sud América. Brasil: Editora CRV.

Guajardo, A. (2016). Construcción de identidades, epistemes y prácticas en Terapia Ocupacional en Latinoamérica. En S. Simó, A. Guajardo, F. Oliver, S. Galheigo, \& S. García (Eds.), Terapias Ocupaciona- les desde el Sur. Derechos Humanos, ciudadanía y participación (pp. 41-62), Santiago de Chile: Editorial Usach.

Martínez Silva, J. (2011). Heidegger y el lenguaje - Posibilidades de superar la concepción instrumental impuesta por la técnica moderna (Tesis de maestría). Universidad de Chile, Chile. Disponible en: http://tesis.uchile.cl/handle/2250/108728

Lukács, G. (1972). Historia y conciencia de clase. La Habana: Editorial de Ciencias Sociales.

Vidal, M. (1990). El hombre Inconcluso: desarrollo personal y clase social. Santiago de Chile: Editorial CINTRAS. 